\title{
NON-Microcomputer Ultra Capacitor Charging System
}

\author{
J. L. Tang ${ }^{1}$, S. Q. Shao ${ }^{1}$ and T. T. Sun ${ }^{1}$ \\ ${ }^{1}$ College of Electrical and Information Engineering ,Southwest Minzu University, Chengdu, \\ Sichuan 610041, China
}

Keywords: Ultra capacitor. NON-microcomputer. CCLV. CVLC.

Abstract. The conventional ultra capacitor charger uses computer to monitor the ultra capacitor's state of charge(SOC) and adopts different strategy to charge the ultra capacitor. It makes the system expensive and the control method is also inconvenient. For the above problems, a non-microcomputer ultra capacitor charging system is designed. The proposed system can achieve two-step charging modes, constant current limiting voltage(CCLV) mode and constant voltage limiting current(CVLC) mode. Finally, the designed charging system is presented at the last of the article.

\section{Introduction}

Renewable energy resources appear to be the one of the most efficient and effective solutions. That is why there is an intimate connection between renewable energy and sustainable development [1-2]. It is urgent to develop renewable energy and related materials. Ultra capacitor is a kind of electrical energy storage device. The advantages of Ultra capacitor are high power density, high efficiency, fast charging and discharging speed, long cycle life, wide operating temperature range and environment friendly [3-4]. It has become an ideal option for high power applications, such as hybrid power systems, regenerative energy systems and instantaneous backup power source.

But charging condition of the ultra capacitor is restricted. The chargers are designed typically around two modes of operation, namely, constant-voltage charging and constant-current charging. The former utilizes a constant voltage source and an equivalent series resistance to control the amount of current that flows into the battery. As soon as the battery voltage is raised to the voltage sources, the converter must limit its current to prevent excessive dissipation. The latter, moreover, keeps the charging current constant until the battery voltage reaches a designated value[5-7]. But it adopts microcomputer to detect the ultra capacitor's SOC that makes the charger expensive and inconvenient. So we design a simple charger.

In section II, the ultra-capacitor's characteristic is described. In section III introduces the designed charging system and the principle of the charging system. Section IV shows the simulation results. Finally, conclusion is made at last.

\section{The ultra capacitor and its charging characteristic introduction}

Ultra capacitor is a high-capacity capacitor with capacitance values much higher than other capacitors, but lower voltage limits. It bridges the gap between electrolytic capacitors and rechargeable batteries and stores 10 to 100 times more energy per unit volume or mass than electrolytic capacitors. The ultra-capacitor can charge and discharge much faster than batteries, and tolerate many more charge and discharge cycles than rechargeable batteries.

Constant voltage charging is also called as constant potential charging used to maintain the same voltage input to the ultra capacitor throughout the charging process, regardless of the ultra capacitor's state of charge. Constant voltage charging provides a high inrush current to the ultra capacitor because of the higher potential difference between ultra capacitor and charger. The charging curve is shown in figure. 1, a.

Constant current charging supplies a relatively constant current, regardless of the ultra capacitor's temperature and state of charge. The charging curve is shown in figure. $1, b$. 


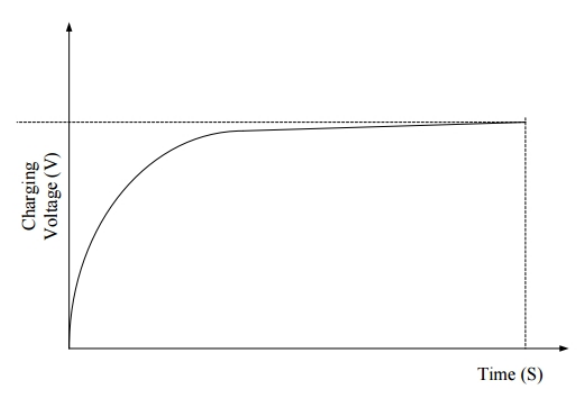

a. Constant voltage charging

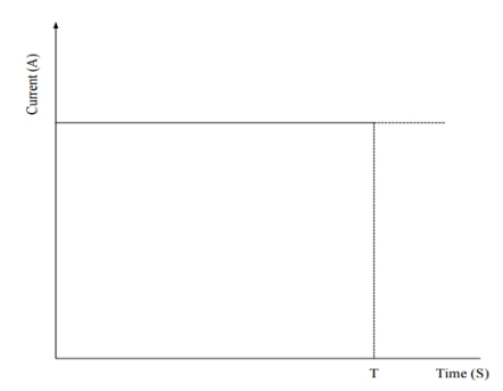

b. Constant current charging

Figure. 1, Ultra capacitor charging curve

\section{The ultra capacitor charging system}

We utilize the buck circuit to charging the ultra-capacitor as shown in figure 2 and by controlling the pulse width of the PWM signal, it can achieve CCLV and CVLC two charging modes under different SOC condition.

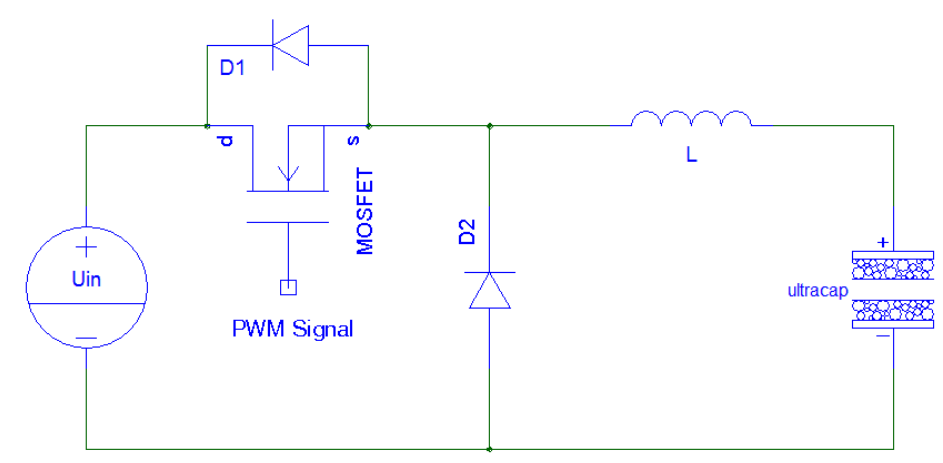

Figure. 2, Ultra capacitor charging circuit

The controlling circuit is shown in figure. $3, V \mathrm{cc}$ is a certain value. According to the characteristics of the operational amplifier, if $V F \neq C F$, one of the amplifier will work at amplification state and the other will work at negative saturation state. So by changing the value of $\mathrm{VF}$ and $\mathrm{CF}$, the charging circuit can work at constant current state or constant voltage state.

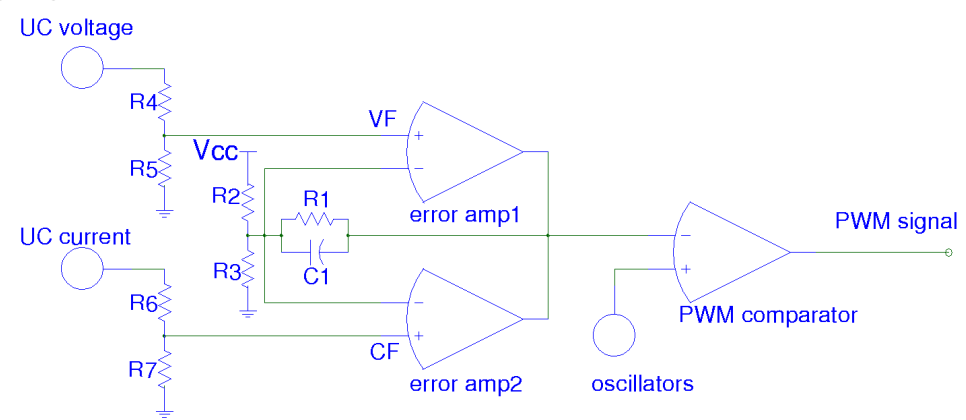

Figure. 3, The control circuit of ultra capacitor charger

The CVLC controlling process is as follow:

(1) $C F<U_{\text {set }}=V F$ : the error amp1 works and the error amp2 doesn't work, the system works in constant voltage mode.

(2) $C F=U_{\text {set }}=V F$ : when the current feedback increases to the setting value, the error amp1 and the error amp2 work together and the system works at the constant voltage and constant current mode. 
(3) $C F>U_{\text {set }}$ : if the load current continues increasing, the error amp2 works and the error amp1 doesn't work. The system works at limiting current mode. As the voltage of the super capacitor rises, the $V F$ increases to the setting value and then the system works at constant voltage mode. The CCLV controlling process is as follow:

(1) $V F<U_{\text {set }}=C F$ : the error amp2 works and the error amp1 doesn't work, the system works in constant current mode.

(2) $V F=U_{\text {set }}=C F$ : when the voltage feedback increases to the set value, the error amp1 and the error amp2 work together and the system works at the critical constant current mode.

(3) $V F>U_{\text {set }}$ : when the voltage sample exceeds the set-value, the error amp1 works and the error amp2 doesn't work. The system works at limiting voltage mode. And the load current increases until the current sample reaching to the setting value, then the controller will work at constant voltage mode. resistor.

The current sampling circuit is shown in figure. 4. Port in- and port in+ connect to sampling

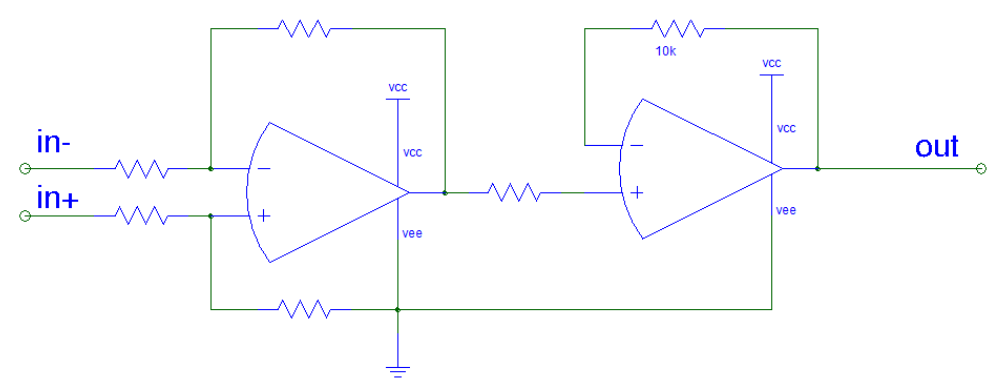

Figure. 4, the current sampling circuit

We choose TL494 as the controlling chip. The system achieves two charging mode according to the ultra capacitor's SOC. The charging circuit is shown at figure, 5. In the figure, TG is CMOS transfer gate and it is controlled by CCLV signal and CVLC signal. When TG1 and TG3 work together, the system works at CCLV mode. When TG2 and TG4 work together, the system works at CVLC mode.

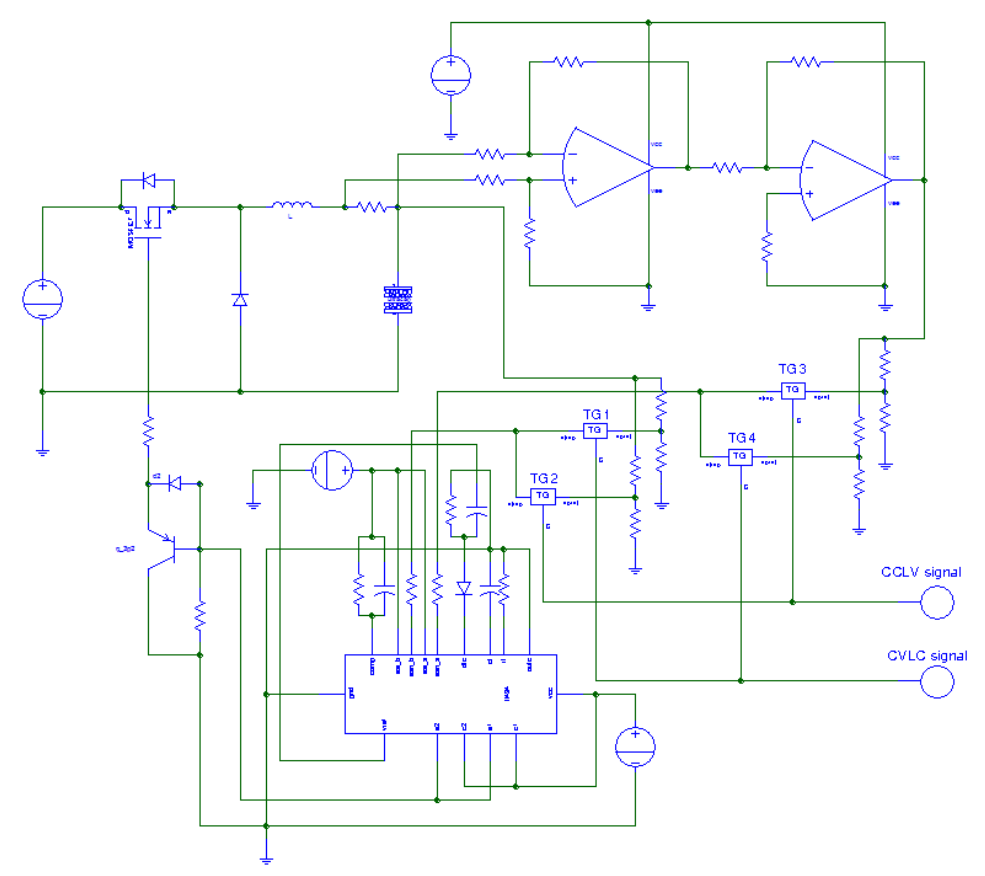

Figure. 5, The realization circuit of ultra capacitor charging system 
The ultra capacitor's state of charge(SOC) detecting circuit is shown in figure. 6. Comparing the instantaneous voltage of super capacitor and its rated voltage, digit selector produces CCLV or CVLC signal.

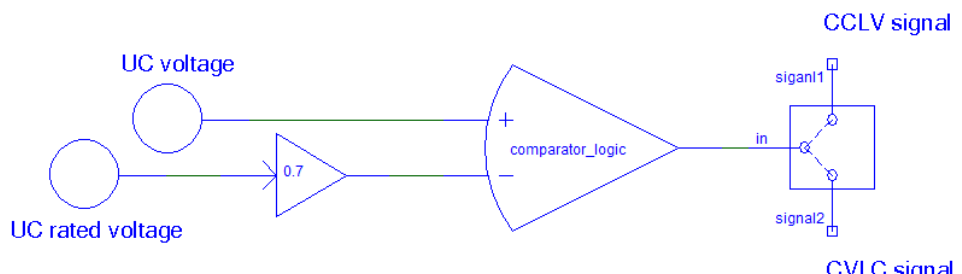

Figure. 6, The ultra capacitor SOC detecting circuit

\section{The ultra capacitor charging system}

The simulation model is implemented in saber sketch. The capacity of the UC is $1400 \mathrm{~F}$, the rated voltage is $2.5 \mathrm{~V}$, the initial voltage is 0 and the number of ultra capacitor in series is 1 . The power source and the simulation result are shown in figure. 7.

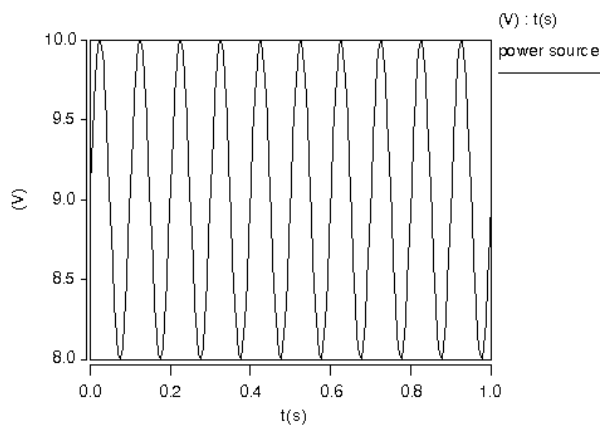

a, power source

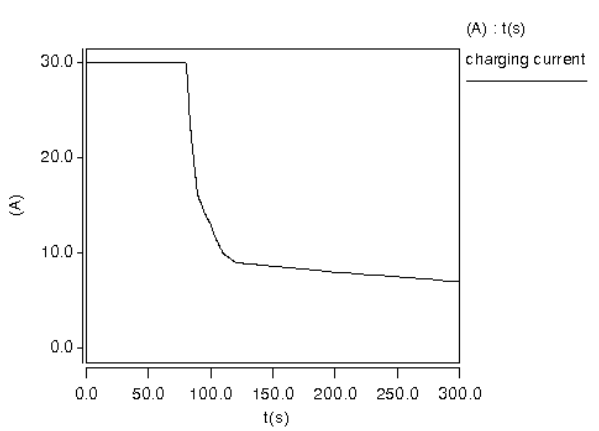

b, charging current

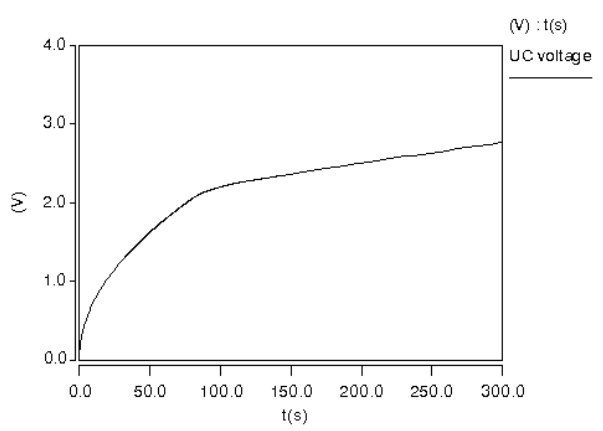

c, UC voltage

Figure. 7, The simulation result: c, power source. a, charging current. b, UC voltage

\section{Conclusions}

A non-microcomputer ultra capacitor charging system is designed. The control principle and the control circuit are also presented. It can charge the ultra capacitor in two modes and achieves two-step charging without microcomputer. Compared to traditional ultra capacitor charger, the cost of the system is cheaper. 


\section{Acknowledgements}

This work was financially supported by the Fundamental Research Funds for the Central Univ ersities(2015NZYQN08), Sichuan Provincial Department of Education Project(No.15ZA0391)

\section{References}

[1] Dincer I, in: Renewable energy and sustainable development: a crucial review[J]. Renewable \& Sustainable Energy Reviews, 2000, 4(2): 157-175.

[2] Omer A M, in: Energy, environment and sustainable development[J]. Renewable \& Sustainable Energy Reviews, 2008, 12(9): 2265-2300.

[ 3] P. Thounthong, S. Raël, B. Davat, in: Fuel cell and supercapacitors for automotive Hybrid Electrical system, ECTI Transactions on Electrical Eng., Electronics, and Communications, vol.3, No.1, 2005, pp.20-30.

[ 4 ] A. Rufer, P. Barrade, in: A supercapacitor-based energy-storage system for elevators with soft commutated interface, IEEE Transactions on Industry Applications, Vol.38, 2002, pp.11511159 .

[ 5 ] N. K. Poon, B. M. H. Pong and C. K. Tse, in: A Constant-Power Battery Charger with Inherent Soft Switching and Power Factor Correction, IEEE Transaction on Power Electronics, Vol. 18, No. 6, 2003, pp. 1262-4269.

[ 6] Hassan F A, in: Power Efficient Battery Charger by Using Constant Current/Constant Voltage Controller[J]. Circuits and Systems, 2012, 03(02): 180-186.

[ 7] Ikeya T, Sawada N, Murakami J I, et al, in: Multi-step constant-current charging method for an electric vehicle nickel/metal hydride battery with high-energy efficiency and long cycle life[J]. Journal of Power Sources, 2002, 105(1):6-12. 